УДК 620.179

Захарченко В. В.

Государственное предприятие «Конструкторское бюро "Южное"» им. М. К. Янгеля. Украина, г. Днепр

\title{
НЕРАЗРУШАЮЩИЙ КОНТРОЛЬ ПРИМЕНЕНИЕ НЕЙРОННЫХ СЕТЕЙ ДЛЯ ОПРЕДЕЛЕНИЯ ГЛУБИНЫ И РАЗМЕРОВ ДЕФЕКТОВ
}

Показана возможность применения термографии, как одного из методов неразрушающего контроля конструкций и материалов с целью выявления дефектов в их структуре. Опробован алгоритм для обработки выходного массива данных термографии. Ключевой особенностью алгоритма является применение нейронных сетей для прогнозирования размеров дефектов. [dx.doi.org/10.29010/082.2]

Ключевые слова: термография; глубина залегания дефекта; нейронная сеть.

\section{Введение}

Неразрушающий контроль методом термографии позволяет, не изменяя свойств материала, изучать распределение температур на поверхности объекта и выявлять скрытые внутренние неоднородности. Термография очень деликатный метод, поэтому применяется даже для изучения предме- тов искусства, археологических находок артефактов, таких как полотна картин и египетские папирусы [1]. Метод позволяет на картинах обнаруживать скрытые слои краски или исправления в древних текстах.

Любая неоднородность в структуре влияет на процесс распространения тепловой энергии (рис. 1). Термография хорошо изучена и стандартизирована 

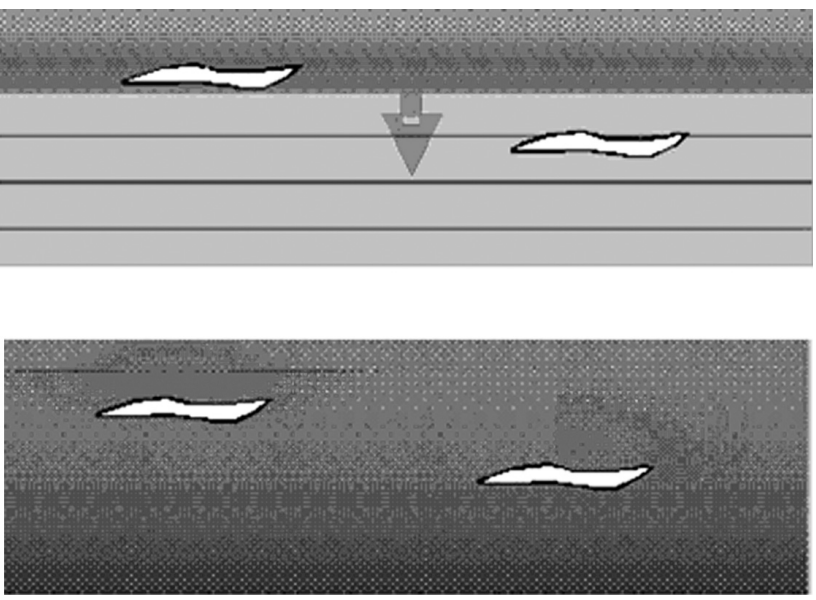

Рис. 1. Влияние дефектов на теплопередачу

американским и отечественным стандартами (ГОСТ 25483-79, ASTM E 2582-07).

Контроль методом термографии заключается в следующем: к объекту прикладывается дозированная и разная по природе нагрузка (тепло, вибрация, вихреток и др.), способная нагреть объект на несколько градусов. Тепловизор записывает нагрев и охлаждение с заданным быстродействием. В результате на рис. 2 приведена схема получения большого массива изображений, каждое изображение фиксирует мгновенное распределение температуры на поверхности объекта контроля.

\section{Постановка задачи}

После проведения термографического контроля на выходе имеется большой трехмерный массив температур. В массиве измеренных температур необходимо выявить аномальные зоны, вызванные нарушением структуры и определить их размеры, такие как площадь и глубину залегания.

\section{Инструменты}

В качестве теплового детектора использовался тепловизор Flir A320, который имел следующие технические характеристики: скорость съемки до 30 Гц, чувствительность $0,3{ }^{\circ} \mathrm{C}$ в диапазоне температур $-20-+120$.

Вычисления производились с помощью библиотеки открытого свободного использования
AForge.NET [4]. AForge.NET имеет более лучшую поддержку функционала для обработки и записи видео файлов по сравнению с Emgu CV. Так же AForge.NET имеет интерфейсы для построения роботизированных систем или автоматов, более богатые возможности для настройки нейронной сети.

\section{Экспериментальная часть}

В качестве объектов исследования в работе изучали: пластину из углепластика, в структуре которой на разную глубину заложены дефекты типа «расслоение» и пластину из углепластика с несквозными отверстиями. Объекты нагревались в течение 45 секунд и остывали в течение 80 последующих секунд. Нагрев и остывание записывался тепловизором Flir A320. В качестве нагревателя использовалась галогеновая лампа мощностью 2 кВт.

Нейронные сети (НС) позволяют решить задачи прогнозирования и классификации, при этом пользователю нет необходимости иметь обширные знания в предметной области. Необходимы лишь знания о подготовке данных и их интерпретации. Существует большое количество разновидностей НС. В данной работе рассматривается регрессионная НС, действующая как апроксиматор. Перед описанием работы архитектуры НС кратко рассмотрим основные понятия.

Нейронная сеть - это система, состоящая из многих простых вычислительных элементов (нейронов), работающих параллельно (рис. 3).

Нейрон представляет собой вычислительную единицу, которая получает информацию, производит над ней простые вычисления и передает ее дальше. Нейроны оперируют числами в диапазоне $[0,1]$ или $[-1,1]$. Для перехода в такой диапазон применяются функции активации.

Функция активащии - это способ нормализации входных данных. То есть, если на входе имеется большое число, то пропустив его через функцию активации, можно получить выход в нужном диапазоне. Функций активации достаточно много: линейные, сигмоиды и гиперболические. Главные их отличия - это диапазон значений.

Как видно из рис. 5 выходная информация представляет собой сумму всех входных данных, умноженных на соответствующие им веса.

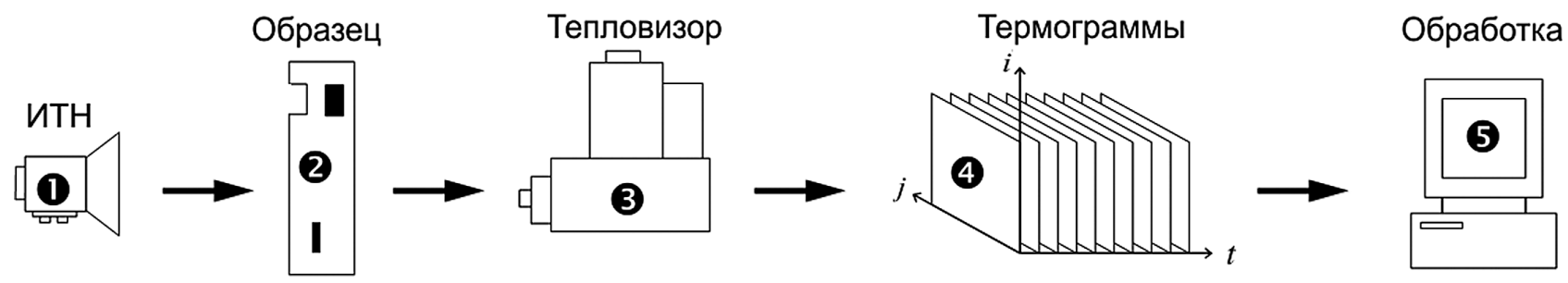

Pис. 2. Схема получения и обработки данных при импульсной термографии 


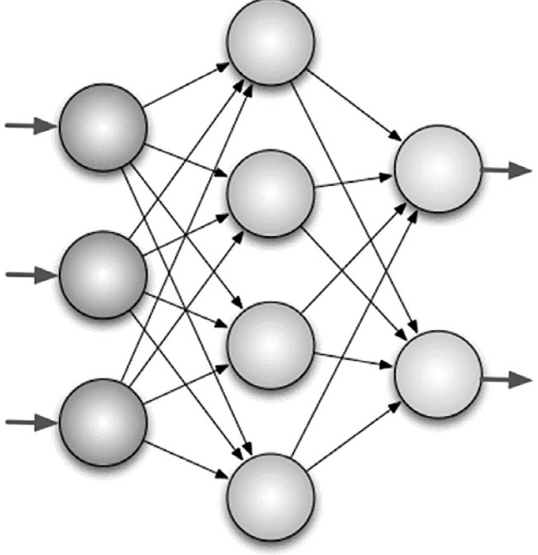

Рис. 3. Нейронная сеть и связи

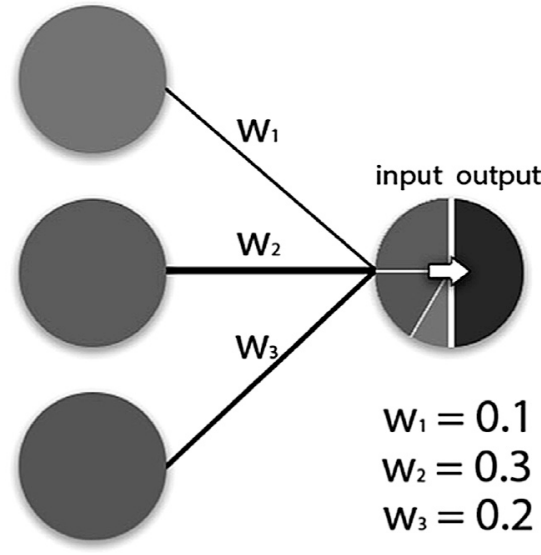

Рис. 4. Пример обработки изображения на одном нейроне

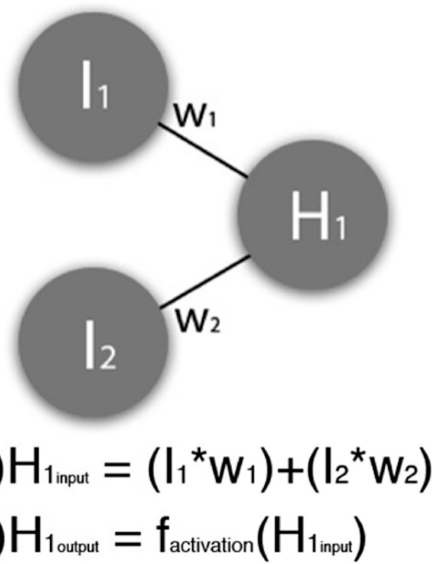

Рис. 5. Пример работы нейронной сети: $I$ - входные нейроны; $H$ - скрытый нейрон; w- веса
В зависимости от задачи строится НС с определенным количеством слоев и нейронов, для нейронов в каждом слое определяют функцию активации, для характеристики связей нейронов определяют веса. Чтобы НС давала правильные прогнозы ее нужно обучать. Обучение заключается в построении или подборе весов в итеративном режиме, для этого существует много алгоритмов [2] обратного распространения, упругого распространения, генетический и др.

НС содержит несколько слоев и стадий. Входной слой делается фиксированным. Для этого из записанного массива температур формируется вектор температур для каждого пикселя длина или вектора зависит от времени контроля. Скрытый слой еще больше ограничивает связи НС, применяя на скрытом слое статистическую процедуру [3] (Анализ Главных Компонент). Нейроны на этом слое представляют собой упорядоченный по убыванию набор по критерию максимальной вариации. Сумма всех компонент на выходе должна давать 100\%-ю вариацию исходного сигнала от одного пикселя. Последний выходной слой содержит только нейроны, которые соответствуют разным пороговым значениям глубин.

Данная НС обучалась по алгоритму обратного распространения. В результате обучения получается хорошая аппроксимация входных значений температур (рис. 7).

Установлено, что основной вклад в вариацию дают первые четыре нейрона в следующем соотношении $84 \%, 15 \%, 1 \%$ и 0,1\%. Таким образом, количество узлов на скрытом слое может быть уменьшено без потери информации. С учетом этого можно модифицировать НС и нейроны на скрытом слое, начиная с пятого компонента нейрона можно заменить случайной величиной с нулевым математическим ожиданием и единичной дисперсией.

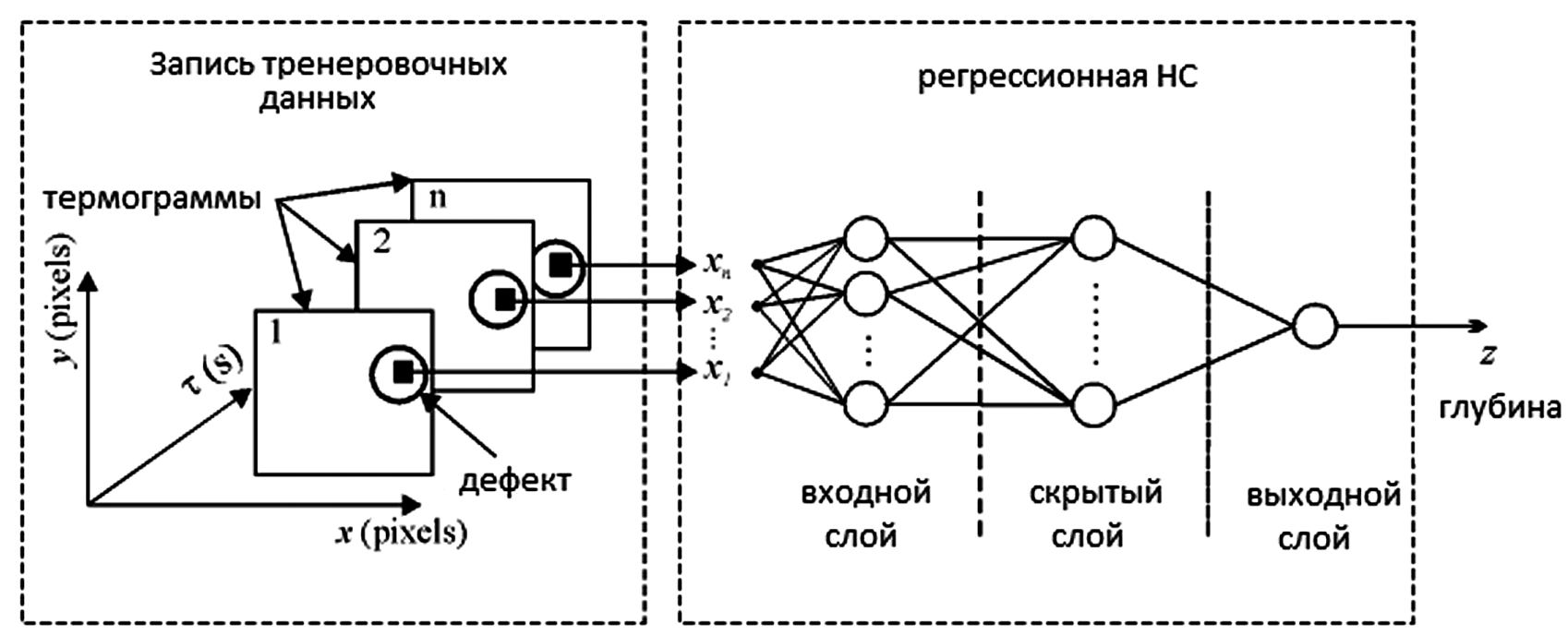

Рис. 6. Архитектура НС 


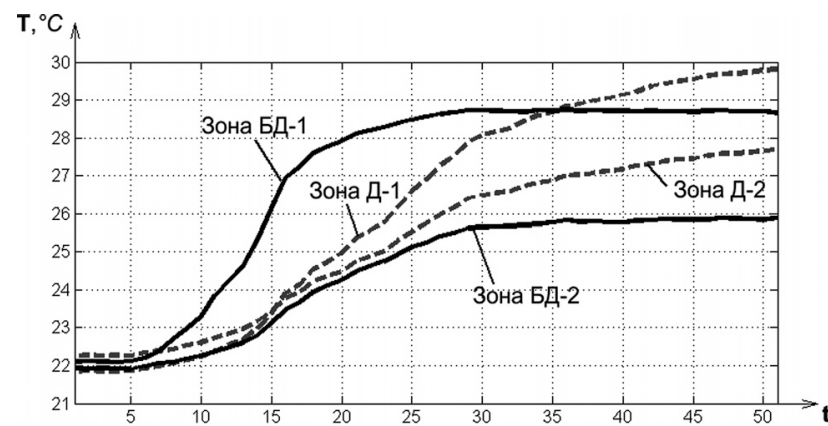

a)

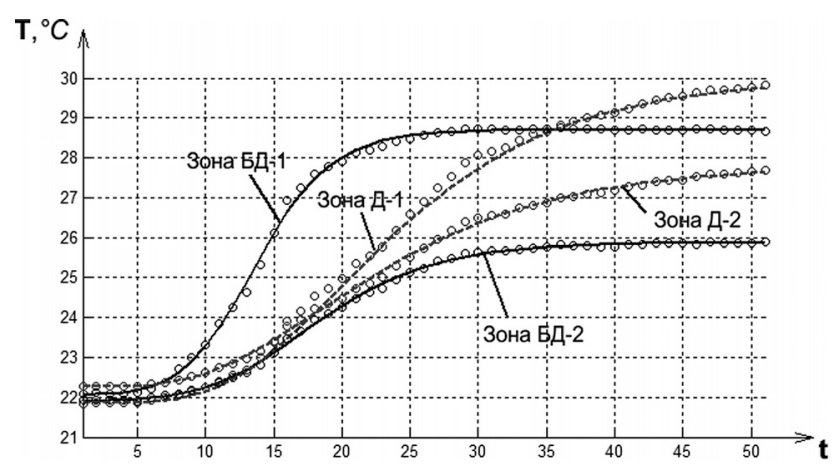

б)

Рис. 7. Аппроксимация входных значений температур: $a-$ исходный вектор; $\sigma$ - вектор на выходе

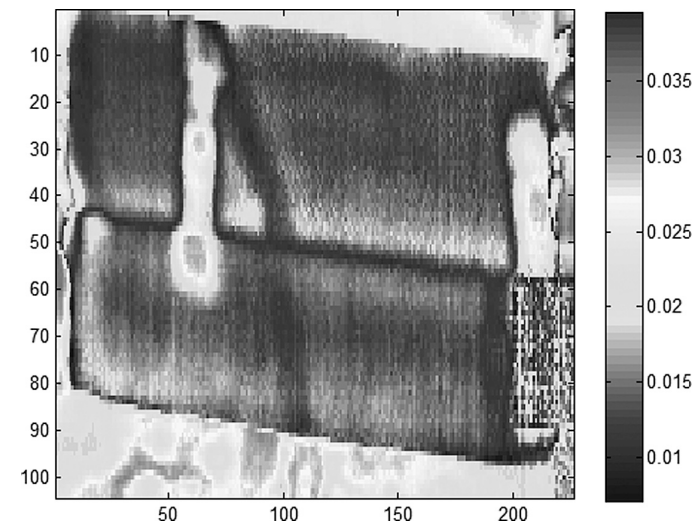

a)

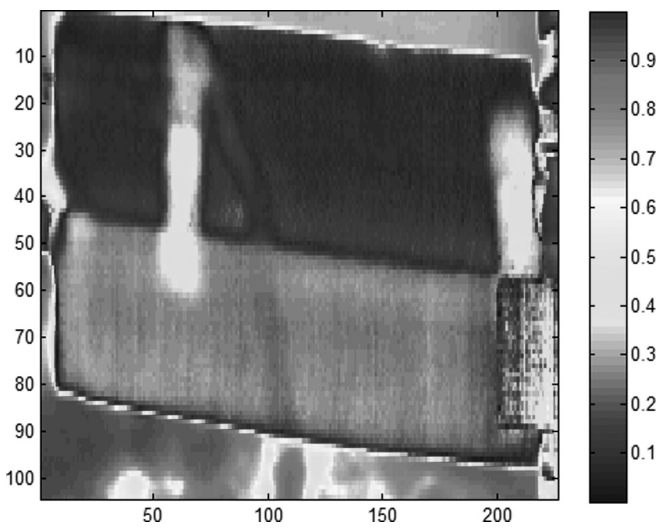

б)

Рис. 8. Результаты модификации НС: $a$ - исходная НС; $\sigma$ - модифицированная НС

\section{Результаты}

1) Применение НС позволило получить более сглаженные последовательности температур (рис. 7).

2) Проведено обучение НС на эталонном образце и ее опробование (рис. 9). НС обучалась на пластине с несквозными отверстиями, а опробовалась на пластине с заложенными дефектами. Учитывая то, что пластина с заложенными дефектами была толще и теплоизолированная, получили некоторые расхождения при определении глубины:

- на глубине 1 мм НС давала значение 0,9 мм,

- на глубине 1,5 мм НС давала значение 1,8 мм,

- на глубине 2,5 мм НС давала значение 2,5 мм,

- на глубине 3,5 мм НС давала значение толщины пластины 3,5 мм.

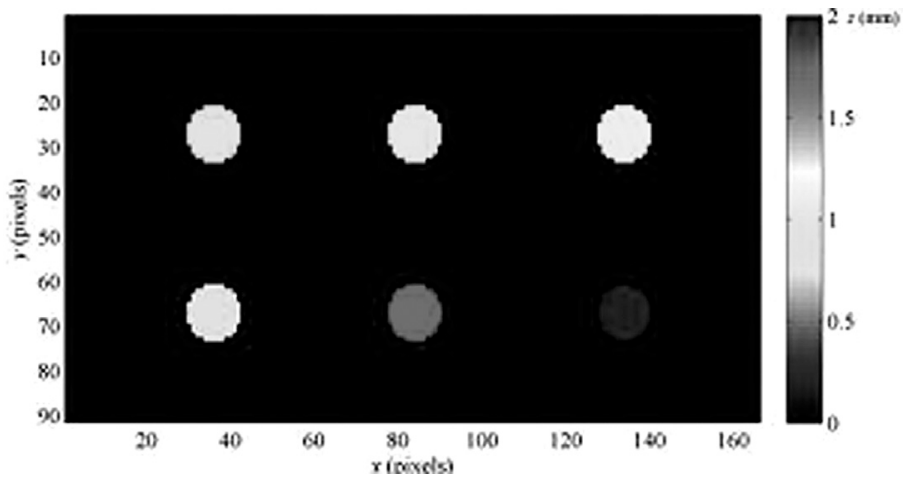

a)

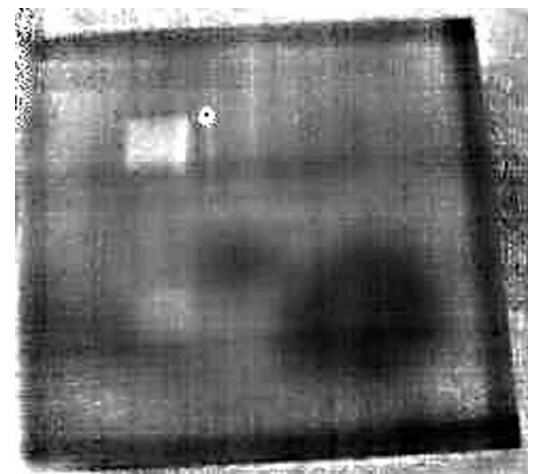

б)

Рис. 9. Результаты обучения и опробования НС:

$a$ - пластина с несквозными отверстиями; 6 - пластина с расслоениями 


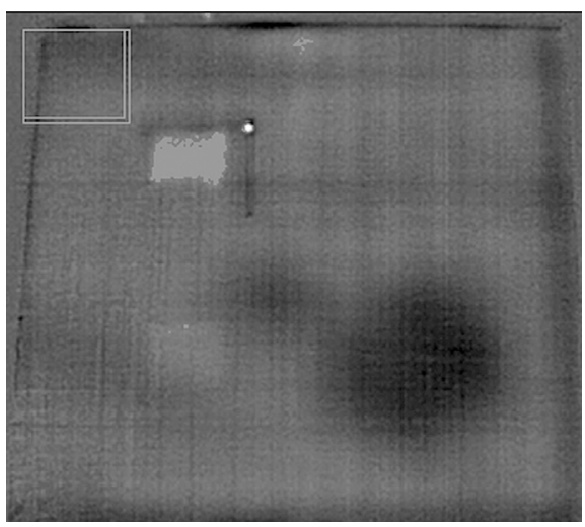

a)

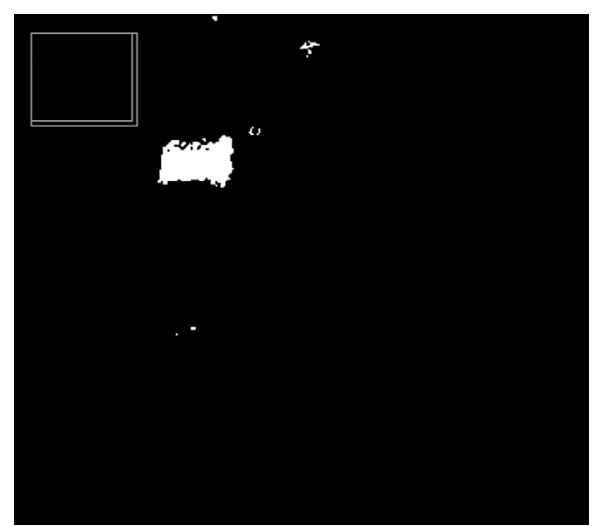

б)

Рис. 10. Результаты кластеризации площади дефекта:

$a$ - выделение кластера дефекта; 6 - бинаризация дефекта по одному пороговому значению

3) Проверено возможность кластеризации дефекта с применением НС (рис. 10). Для обученной НС не представляет никакой трудности выделить площадь дефекта. Достаточно для функции активации на последнем слое НС определить порог и НС начнет выполнять бинаризацию.

\section{Литература}

[1] Document flash thermography, Cory A. Larsen, Utah State University 2011, p. 147.
[2] Data approximation by artificial neural network, T.V. Phylatova, Tomsk 2004, p. 153-158.

[3] Characterization of material defects using active THERMOGRAPHY and an artificial neural network, Sebastian Dudzik, Częstochowa University of Technology 2013, Metrol. Meas. Syst., Vol. XX (2013), No. 3, pp. 491-500.

[4] Open Source .NET Libraries for Image Processing, Recognition and Computer Vision, Stefan Toth, Zilina University Slovakia 2015, Vol XVI, No 2, pp 23-30.

\section{Zakharchenko V.V.}

Yuzhnoye, State-owned Design Office named after M. K. Yangel. Ukraine, Dnipro

\section{USING OF ARTIFICIAL NEURAL NETWORK FOR EVALUATING DEPTH AND SIZE OF THE DEFECTS IN NON-DESTRUCTIVE EVALUATION}

In this paper are dicused ability one of non-destructive testing method the thermography, its main purpose to find out flaws in material structure. As will be shown out processing algorithm of thermography output data. The key feature of algorithm is neural network for prediction size and depth of flazes. [dx.doi.org/10.29010/082.2]

Keywords: thermography; depth of defect; neural network.

\section{References}

[1] Document flash thermography, Cory A. Larsen, Utah State University 2011, p. 147.

[2] Data approximation by artificial neural network, T.V. Phylatova, Tomsk 2004, p. 153 - 158.

[3] Characterization of material defects using active THERMOGRAPHY and an artificial neural network, Sebastian Dudzik, Częstochowa University of Technology 2013, Metrol. Meas. Syst., Vol. XX (2013), No. 3, pp. 491—500.

[4] Open Source .NET Libraries for Image Processing, Recognition and Computer Vision, Stefan Toth, Zilina University Slovakia 2015, Vol XVI, No 2, pp $23-30$. 